\title{
Improvement on Turn-on Speed of Substrate-Triggered SCR Device by Using Dummy-Gate Structure for On-Chip ESD Protection
}

\author{
Kuo-Chun Hsu and Ming-Dou Ker \\ Nanoelectronics and Gigascale Systems Laboratory, Institute of Electronics, National Chiao-Tung University \\ 1001 Ta-Hsueh Road, Hsinchu, Taiwan \\ Tel: (+886)-3-5131573, Fax: (+886)-3-5715412, E-mail: mdker@ieee.org
}

\section{Introduction}

An excellent ESD protection device must have high enough ESD robustness and faster turn-on speed to effectively protect the gate oxide of input stage from ESD overstress. With the best area efficiency, SCR can sustain highest ESD level in a smallest layout area, as comparing to other ESD protection devices. So, SCR had been used as on-chip ESD protection for a long time [1]. But, SCR still has a higher switching voltage $(20 \mathrm{~V})$ in CMOS technology, which is generally greater than the gate-oxide breakdown voltage of the input stage. Some reports had presented the solutions to overcome this issue [2], including the ST_SCR device [3].

In this work, a novel dummy-gate structure is used to block the shallow trench isolation (STI) region in SCR device and to further enhance its turn-on speed. With suitable ESD-detection circuit [3], the ST_SCR with dummy-gate structure is designed to be kept off during the normal circuit operating conditions, and to be quickly triggered on during the ESD-zapping conditions. Therefore, the ultra-thin gate oxide in deep submicron CMOS processes can be effectively protected by the ST_SCR with dummy-gate structure.

\section{SCR Device With Dummy-Gate Structure}

In deep submicron CMOS processes, the diffusion regions are isolated by STI. With the dummy-gate structures, the STI regions between the diffusions in SCR device are blocked. The ST_SCR device with STI is shown in Fig. 1(a), whereas the proposed ST_SCR device with dummy-gate structure is shown in Fig. 1(b). In a typical $0.25-\mu \mathrm{m}$ CMOS process, the depth of STI is about $\sim 0.4 \mu \mathrm{m}$, but the junction depth of $\mathrm{P}+/ \mathrm{N}+$ diffusion is only about $\sim 0.18 \mu \mathrm{m}$. The deeper STI region in SCR device causes a longer current
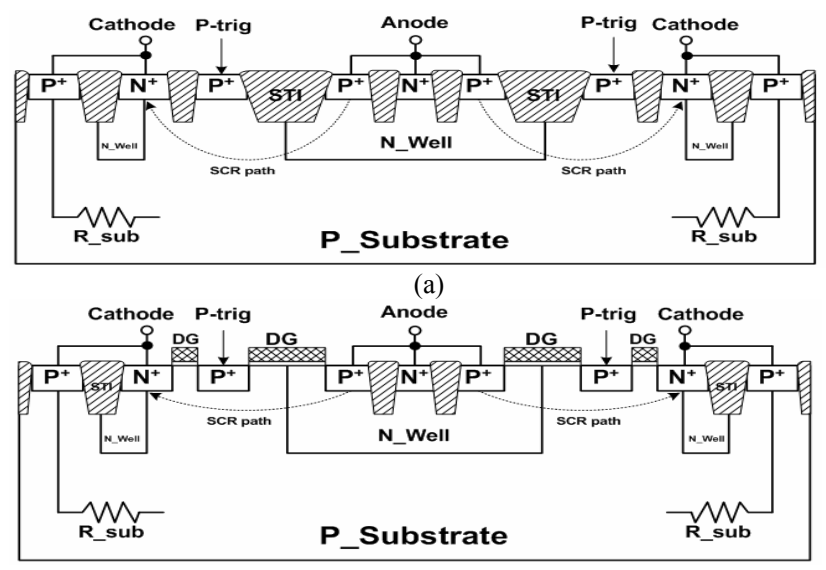

(b)

Fig. 1 Device structures of the substrate-triggered SCR (ST_SCR) with (a) STI structure and (b) dummy-gate structure. path from the anode to the cathode, which also leads to a slower turn-on speed. The ESD current path in the ST_SCR with dummy-gate structure indicated by the dashed line in Fig. 1(b) is shorter than that in ST_SCR with STI in Fig. 1(a), because the dummy-gate structure is used to block the $\mathrm{STI}$ region in SCR device. The inserted $\mathrm{P}+$ diffusions are connected out as the p-trigger node of the ST_SCR. When a trigger current is applied into the trigger node, the ST_SCR can be triggered on into its latching state. For ESD protection purpose, the corresponding ESD-detection circuit has to be designed to control the turn-on of this ST_SCR.

\section{Experimental Results \\ Device Characteristics}

The two ST_SCR devices with STI and dummy-gate structures have been fabricated with the same layout area in a fully-silicided $0.25-\mu \mathrm{m}$ CMOS process. The dependences of switching voltages (Vt1) of ST_SCRs with STI and dummy-gate structures on the substrate-triggered current are compared in Fig. 2 and the inset of Fig. 2 is the DC I-V curves of ST_SCR with dummy-gate structure under different substrate-triggered currents. When the substratetriggered current at the p-trigger node is increased from $0 \mathrm{~mA}$ to $6 \mathrm{~mA}$, the Vt1 of ST_SCR with STI is reduced from $\sim 22 \mathrm{~V}$ to $\sim 7 \mathrm{~V}$, whereas the $\mathrm{Vt1}$ of ST_SCR with dummy-gate structure is reduced from $\sim 18 \mathrm{~V}$ to $\sim 3 \mathrm{~V}$. If the trigger current is continually increased, the Vt1 of both ST_SCRs will be nearly reduced to their holding voltages $(\sim 1.3 \mathrm{~V})$. This result has proven that the $\mathrm{Vt1}$ of both ST_SCRs with STI and dummy-gate structure can be significantly reduced by the substrate-triggered technique. Moreover, the Vt1 of ST_SCR with dummy-gate structure can be further reduced lower than that of ST_SCR with STI under the same trigger current. This is related to the current gain $(\beta)$ of parasitic bipolar transistor in SCR structure,

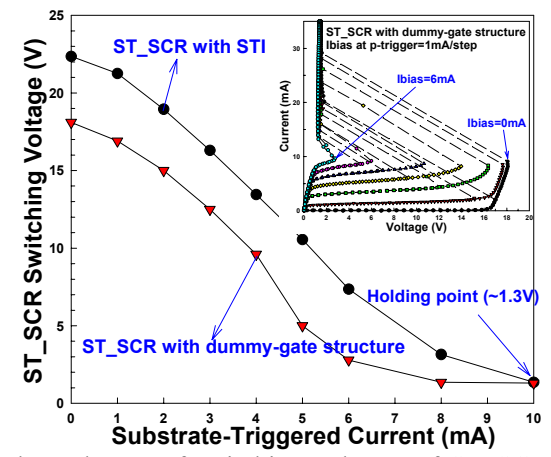

Fig. 2 The dependences of switching voltages of ST SCR devices with STI and dummy-gate structure on the substrate-triggered current. The inset is the DC I-V curves of ST_SCR with dummy-gate structure under different substrate-triggered currents. 
which will be discussed later. The SCR device with lower Vt1 can clamp ESD overstress more quickly to effectively protect the thinner gate oxide of input circuits.

\section{Turn-on Speed}

The turn-on time of ST_SCR is defined as the time for ST_SCR entering into its latching state. The measured turn-on times for ST SCRs with STI and dummy-gate structure are shown in Figs. 3(a) and 3(b), respectively. The inset of Fig. 3(a) is the measurement setup. From Fig. 3(a), the turn-on time of ST_SCR with STI is reduced from $35 \mathrm{~ns}$, $20 \mathrm{~ns}$, to $11.2 \mathrm{~ns}$, while the ST SCR is triggering by the voltage pulse of $1.5 \mathrm{~V}, 2 \mathrm{~V}$, and $\overline{4 V}$ with 10 -ns rise time into the trigger node. Moreover, from Fig. 3(b), the turn-on time of ST_SCR with dummy-gate structure is further reduced from $25.4 \mathrm{~ns}, 13.6 \mathrm{~ns}$, to $9.8 \mathrm{~ns}$ under the same measurement conditions as that of Fig. 3(a). The relations between the turn-on time and the triggering pulse voltage of ST_SCRs with STI and dummy-gate structure are compared in Fig. 4. The turn-on time of ST_SCR with dummy-gate structure can be effectively shortened, as comparing to the ST_SCR with STI. For CMOS IC applications with ultra-thin gate oxide, the ST_SCR with dummy-gate structure can be designed to protect the core circuits from latent damages or

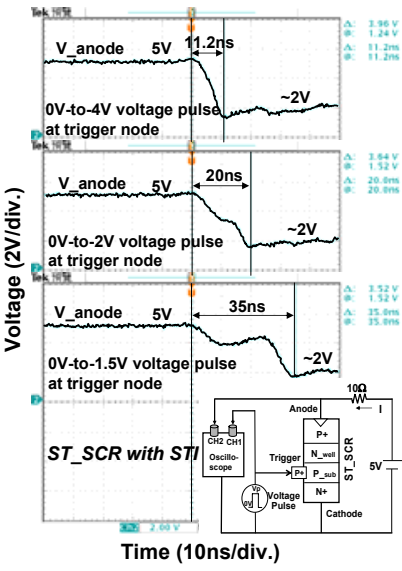

(a)

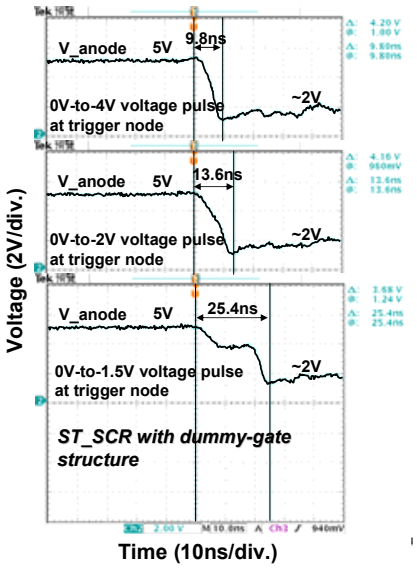

(b)
Fig. 3 Measurement on the turn-on time of ST_SCR under different voltage pulses. The measured voltage waveforms on the anode of the ST_SCR with (a) STI, and (b) dummy-gate structure, while the ST_SCR is triggering by the voltage pulse of $1.5 \mathrm{~V}, 2 \mathrm{~V}$, and $4 \mathrm{~V}$ with 10 -ns rise time into the trigger node. The inset in (a) is the measurement setup.

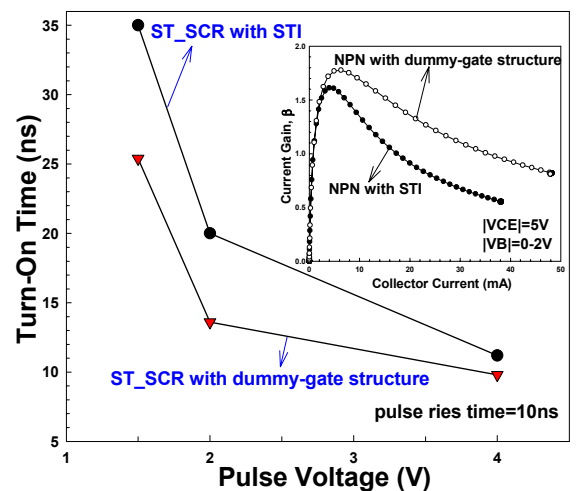

Fig. 4 The relations between the turn-on time and the triggering pulse voltage of ST_SCR with STI and dummy-gate structure. The inset is the dependence of current gains of the NPN bipolar transistors in the ST_SCR devices on its collector current. failure more efficiently than the ST_SCR with STI. For analyzing the different characteristics of ST_SCRs, the dependence of current gains of NPN bipolar transistors in the ST_SCR with STI and dummy-gate structure on the collector current under the measured conditions of $|\mathrm{VCE}|=5 \mathrm{~V}$ and $|\mathrm{VB}|=0-2 \mathrm{~V}$ is shown in the inset of Fig. 4. In the inset of Fig. 4, the current gain of NPN in the ST_SCR with dummy-gate structure is higher than that of NPN in the ST_SCR with STI. The Vt1 of ST_SCR device is in inverse proportion to the current gain [4], so the ST_SCR with dummy-gate structure has the lower Vt1. In addition, substrate bias used to trigger the NPN transistor in the ST_SCR device has significant effect to further reduce Vt1 and turn-on time of ST_SCR with dummy-gate structure.

The dependence of turn-on time of ST_SCR with dummy-gate structure on rise time of voltage pulse under different substrate bias conditions is also measured and shown in Fig. 5. When a 4-V substrate pulse with rise time of $5 \mathrm{~ns}$ is applied to the p-trigger node, the turn-on time of the ST_SCR can be shortened to $6.6 \mathrm{~ns}$. So, the turn-on speed of ST_SCR with dummy-gate structure can trace the rise time of $\overline{E S D}$ events to discharge the ESD current faster.

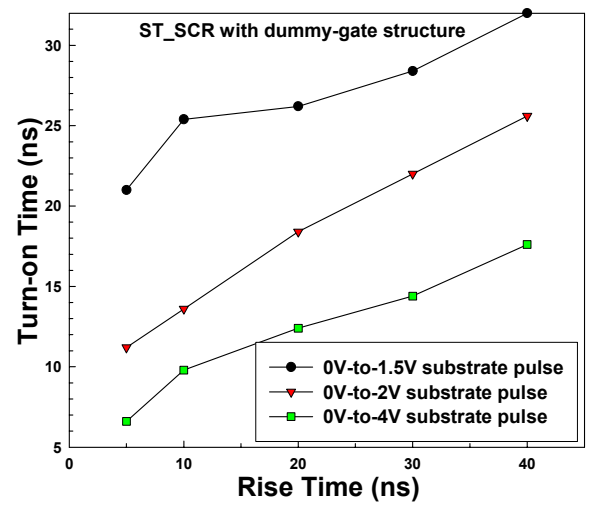

Fig. 5 The dependence of turn-on time of ST_SCR with dummy-gate structure on rise time of voltage pulse under different substrate bias conditions.

\section{Conclusions}

The novel dummy-gate structure in SCR device with substrate-triggered design has been successfully investigated in a $0.25-\mu \mathrm{m}$ salicided CMOS process. The dummy-gate structure can indeed reduce the switching voltage and improve turn-on speed of ST_SCR device without increasing any cost, as comparing to the traditional SCR structure. With an improved turn-on speed, the proposed ST_SCR with dummy-gate structure can effectively protect the ultra-thin gate oxide against ESD damage in deep submicron CMOS integrated circuits.

\section{References}

[1] M.-D. Ker, "Electrostatic discharge protection circuits in CMOS IC's using the lateral SCR devices: an overview," in Proc. IEEE Int. Conf. on Electronics Circuits and Systems, 1998, pp. 325-328

[2] A. Chatterjee and T. Polgreen, "A low-voltage triggering SCR for on-chip ESD protection at output and input pads," IEEE EDL, vol. 12, pp. 21-22, 1991.

[3] M.-D. Ker and K.-C. Hsu, "Substrate-triggered SCR device for on-chip ESD protection in fully silicided subquarter-micrometer CMOS process," IEEE T-ED, vol. 50, pp. 397-405, 2003.

[4] V. Gupta, et al., "ESD-related process effects in mixed-voltage sub-0.5 $\mu \mathrm{m}$ technologies," in Proc. EOS/ESD Symp., 1998, pp. 161-169. 